\title{
Glomerular Capillary and Endothelial Cell Injury is Associated with the Formation of Necrotizing and Crescentic Lesions in Crescentic Glomerulonephritis
}

\author{
Emiko Fujita ${ }^{1}$, Kiyotaka Nagahama ${ }^{2}$, Akira Shimizu $^{2}$, Michiko Aoki ${ }^{1,2}$, \\ Seiichiro Higo ${ }^{1,2}$, Fumihiko Yasuda ${ }^{1,2}$, Akiko Mii ${ }^{1}$, Megumi Fukui ${ }^{1}$, \\ Tomohiro Kaneko ${ }^{1}$ and Shuichi Tsuruoka ${ }^{1}$ \\ ${ }^{1}$ Department of Nephrology, Nippon Medical School \\ ${ }^{2}$ Department of Analytic Human Pathology, Nippon Medical School
}

\begin{abstract}
Background: The associations of glomerular capillary and endothelial injury with the formation of necrotizing and crescentic lesions in cases of crescentic glomerulonephritis (GN) have not been evaluated in detail.
\end{abstract}

Methods: Glomerular capillary and endothelial cell injury were assessed in renal biopsy specimens of crescentic GN, including those from patients with anti-neutrophil cytoplasmic autoantibodies (ANCA)associated GN (n=45), anti-glomerular basement membrane (GBM) GN (n=7), lupus GN (n=21), and purpura GN $(n=45)$ with light and electron microscopy and immunostaining for CD34.

Results: In ANCA-associated GN, anti-GBM GN, lupus GN, and purpura GN, almost all active necrotizing glomerular lesions began as a loss of individual CD34-positive endothelial cells in glomerular capillaries, with or without leukocyte infiltration. Subsequently, necrotizing lesions developed and were characterized by an expansive loss of CD34-positive cells with fibrin exudation, GBM rupture, and cellular crescent formation. With electron microscopy, capillary destruction with fibrin exudation were evident in necrotizing and cellular crescentic lesions. During the progression to the chronic stage of crescentic GN, glomerular sclerosis developed with the disappearance of both CD34-positive glomerular capillaries and fibrocellular-to-fibrous crescents. In addition, the remaining glomerular lobes without crescents had marked collapsing tufts, a loss of endothelial cells, and the development of glomerular sclerosis.

Conclusions: The loss of glomerular capillaries with endothelial cell injury is commonly associated with the formation of necrotizing and cellular crescentic lesions, regardless of the pathogeneses associated with different types of crescentic GN, such as pauci-immune type ANCA-associated GN, anti-GBM GN, and immune-complex type GN. In addition, impaired capillary regeneration and a loss of endothelial cells contribute to the development of glomerular sclerosis with fibrous crescents and glomerular collapse. (J Nippon Med Sch 2015; 82: 27-35)

Key words: CD34, crescentic glomerulonephritis, endothelial cell injury, glomerular necrosis, microscopic polyangiitis

\section{Introduction}

Rapidly progressive glomerulonephritis (RPGN) is a syndrome characterized by the rapid loss of renal function, often accompanied by oliguria or anuria, with features of glomerulonephritis (GN), including hematuria and glomerular proteinuria ${ }^{1,2}$. Aggressive GN that causes RPGN is usually associated with extensive glomerular crescent formation ${ }^{3,4}$. For this reason, the clinical term "RPGN" is sometimes used interchangeably with the pathological term "crescentic GN." ${ }^{\prime 1,2}$. The rupture of

Correspondence to Emiko Fujita, MD, PhD, Department of Nephrology, Nippon Medical School, 1-1-5 Sendagi, Bunkyo-ku, Tokyo 113-8603, Japan

E-mail: e-suga@nms.ac.jp

Journal Website (http://www.nms.ac.jp/jnms/) 
glomerular capillary walls allows fibrin, plasma proteins, inflammatory mediators, and leukocytes to enter Bowman's space, where they induce the proliferation of epithelial cells in Bowman's capsule and the infiltration of macrophages, which together produce cellular crescents. Crescentic GN can be classified into 3 major clinical categories: immune-complex type GN, anti-glomerular basement membrane (anti-GBM) GN, and pauci-immune type GN, which often is associated with antineutrophil cytoplasmic autoantibodies (ANCA) (1,2. $^{1}$.

The glomerulus is a well-developed capillary network. The glomerular capillaries are lined by a thin, fenestrated endothelium that is critically involved in controlling the vascular tone, coagulation, inflammation, and homeostasis of glomerular functions ${ }^{5,6}$. The injury of glomerular capillaries and endothelial cells may mediate the progression of crescentic GN. However, glomerular endothelial cell injury and glomerular capillary injury have not been evaluated pathologically in detail in cases of crescentic GN. Moreover, the precise mechanisms that lead to crescentic glomerular lesions in GN remain unclear.

In the present study, we first determined which endothelial cell marker was best for morphologically detecting glomerular capillaries in sections of formalin-fixed, paraffin-embedded tissue obtained at routine renal biopsy. We next evaluated the morphological alterations of the glomerular capillaries in crescentic GN to clarify the correlations between the presence of injuries to glomerular capillaries and endothelial cells and the formation of necrotizing and crescentic glomerular lesions in crescentic GN.

\section{Materials and Methods}

\section{Renal Biopsy Cases of Crescentic GN}

The renal biopsy specimens from the Department of Pathology, Nippon Medical School, obtained from 2008 through $2013(n=1,031)$ were reviewed. Renal biopsies were performed with informed consent when unexplained nontransient hematuria, proteinuria, or renal dysfunction was present, alone or in combination. The pathological diagnosis of crescentic GN was confirmed with light, immunofluorescence, and electron microscopic findings. We selected renal biopsy cases of crescentic GN, including ANCA-associated microscopic polyangiitis ( $\mathrm{n}=$ 45), anti-GBM GN ( $n=7)$, lupus GN ( $n=21)$, and HenochSchönlein purpura nephritis (HSPN) $(n=45)$. The age, sex, blood pressure, the presence of hematuria (red blood cells $/ \times 400$ high-power field) or proteinuria (g/day), and serum creatinine levels $(\mathrm{mg} / \mathrm{dL})$ at the time of biopsy were reviewed.

\section{Pathological Examination}

Kidney biopsy specimens that were fixed in $20 \%$ buffered formalin and embedded in paraffin were used for light microscopic examinations. Hematoxylin and eosin (H\&E), periodic acid-Schiff (PAS), Masson's trichrome, and periodic acid-methenamine silver (PAM) staining was performed for light microscopic examination ${ }^{7,8}$. Immunofluorescence for immunoglobulin (Ig) G, IgM, and IgA and complement components (C1q, C3, and C4) was examined using frozen biopsy tissues ${ }^{7,8}$. Electron microscopic examination was performed with tissues fixed in $2.5 \%$ glutaraldehyde, postfixed in $1 \%$ osmium tetroxide, and embedded in Epon ${ }^{7,8}$. Ultrathin sections were stained with uranyl acetate and lead citrate and then examined with a electron microscope (H7500, Hitachi, Ibaraki, Japan).

For immunohistochemical studies to detect glomerular capillaries, the formalin-fixed, paraffin-embedded biopsy specimens were stained with the standard avidin-biotinperoxidase complex technique. The primary antibodies used included: 1) a monoclonal mouse anti-human CD34 antibody (NU-4A1, Nichirei Bioscience, Tokyo, Japan), 2) a monoclonal mouse anti-human CD31 antibody (M0823, DAKO, Glostrup, Denmark), 3) a polyclonal rabbit antihuman von Willebrand factor (vWF) (factor VIII antigen) antibody (A0082, DAKO), 4) a polyclonal rabbit antihuman anti-thrombomodulin (TM) antibody (M0617, DAKO), 5) a monoclonal mouse anti-human nestin antibody (MAB1259, R\&D Systems, Minneapolis, MN, USA), 6) a monoclonal mouse anti-human CD146 antibody (Novocastra, NCL-146, Newcastle, United Kingdom), and 7) a polyclonal rabbit anti-human caveolin-1 antibody (Santa Cruz Biotechnology, Santa Cruz, CA, USA). To optimize the detection of CD34, vWF, and TM, tissue sections were heated in a microwave oven for 10 minutes in $0.01 \mathrm{~mol} / \mathrm{L}$ sodium citrate $(\mathrm{pH}$ 6.0) and incubated with $0.1 \%$ protease for 10 minutes and $0.1 \%$ pepsin for 15 minutes before incubation with the primary antibody.

Detection of Glomerular Capillary and Endothelial

\section{Cell Injuries}

The injury of glomerular capillaries and endothelial cells in crescentic GN was assessed with light and electron microscopy and with immunohistochemical staining for CD34. The loss of glomerular capillaries and endothelial cells was evaluated in the formation of necrotizing glomerular lesions, and in cellular, fibrocellular, and fibrous crescents, with or without glomerular collapse and glomerular sclerosis. 

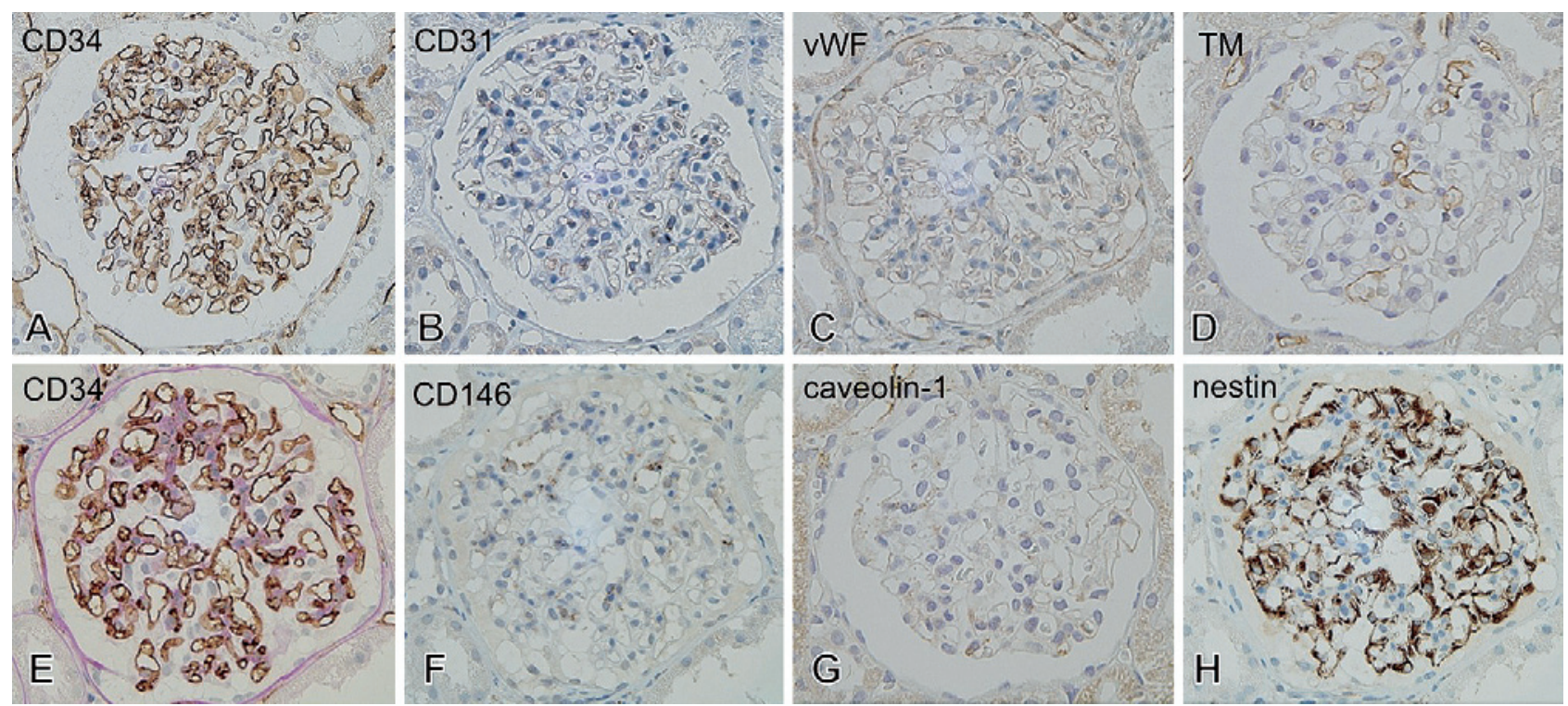

Fig. 1 Immunohistochemical staining of glomerular endothelial cells

Immunohistochemistry for the detection of glomerular capillaries in normal control glomeruli (A-D), or in glomeruli with minor glomerular abnormalities $(\mathrm{E}-\mathrm{H})$ in formalin-fixed paraffin-embedded tissue sections (A, E: CD34 staining; B: CD31 staining, C: von Willebrand factor (vWF) (Factor VIII) staining; D thrombomodulin staining (TM); F: CD146 staining; J: caveolin-1 staining and $\mathrm{K}$ : nestin staining; $\times 600)$.

In normal glomeruli and glomeruli with minor glomerular abnormalities, CD34 immunostaining could detect the glomerular capillary network. However, immunostaining for CD31, vWF, thrombomodulin, CD146, caveolin-1 and nestin could not detect glomerular capillaries clearly using our techniques.

\section{Results}

\section{CD34 Immunostaining to Detect Glomerular Capillar-} ies

In normal glomeruli and in glomeruli with minor glomerular abnormalities, immunostaining for CD34 indicated glomerular capillaries (Fig. 1). However, immunostaining for CD31, vWF, TM, CD146, and caveolin1 , which are common markers of endothelial cells, could not indicate glomerular capillaries when our techniques were used, because immunostaining was weak or undetectable on endothelial cells. In addition, nestin was expressed on glomerular epithelial cells and on endothelial cells. We, therefore used immunostaining for CD34 to detect morphological changes in glomerular capillaries in glomeruli. To observe the correlation between the glomerular capillaries and GBM, PAS stain was added after CD34 immunostaining in the same section.

\section{Characteristics of Patients}

The cases of crescentic GN ( $n=118)$ included 45 cases of ANCA-associated GN, 7 cases of anti-GBM GN, 21 cases of lupus GN, and 45 cases of HSPN. The characteristics of the patients are shown in Table 1. All patients with crescentic GN had massive hematuria (median >100 RBC cells/high-power field) and proteinuria (more than $1 \mathrm{~g}$ / day). The patients with lupus GN had a nephrotic range of proteinuria $(3.3 \pm 1.7 \mathrm{~g} /$ day), because cases of lupus GN included cases of class III or class IV and class V. The patients with ANCA-associated GN were older than patients with other types of crescentic GN. The cases of anti-GBM GN were characterized by a higher male ratio, higher blood pressure, higher serum creatinine levels, and higher rates of cellular and fibrocellular crescent formation than those in cases of other types of crescentic GN.

\section{CD34-positive Glomerular Capillaries in Crescentic GN \\ Crescentic GN was commonly classified as the pauci-} immune type GN, such as ANCA-associated GN, antiGBM GN, and immune-complex type GN, including lupus GN and HSPN. Even in the cases in different categories, crescentic GN had common pathological findings, such as necrotizing and cellular crescentic glomerular lesions in the acute and active phases.

Focal necrotizing glomerular lesions were found with rupture of the GBM and no obvious cellular infiltrates in ANCA-associated GN (Fig. 2A). Endothelial cells positive for CD34 were lost in the necrotizing lesions, and the destruction of glomerular capillaries was noted with rupture of the GBM (Fig. 2E). With electron microscopy, exu- 
Table 1 Clinical characteristics of patients with crescentic GN

\begin{tabular}{lcccc}
\hline & ANCA-associated GN & Anti-GBM GN & Lupus GN & HSPN \\
\hline cases & $\mathrm{n}=45$ & $\mathrm{n}=7$ & $\mathrm{n}=21$ & $\mathrm{n}=45$ \\
Age (years) & $66.5 \pm 11.9$ & $52.1 \pm 20.2$ & $41.9 \pm 19.3$ & $31.2 \pm 26.1$ \\
Male (\%) & $53.3 \%$ & $71.4 \%$ & $47.6 \%$ & $53.3 \%$ \\
Systolic BP (mm Hg) & $130.6 \pm 10.3$ & $153.5 \pm 10.6$ & $140.5 \pm 17.8$ & $133.3 \pm 25.2$ \\
Diastolic BP (mm Hg) & $74.6 \pm 9.2$ & $71.0 \pm 12.7$ & $84.8 \pm 14.8$ & $72.1 \pm 12.7$ \\
Serum Cr (mg/dL) & $2.6 \pm 2.4$ & $9.0 \pm 2.1$ & $1.6 \pm 1.0$ & $1.5 \pm 1.8$ \\
Urine Protein (mg/dL) & $1.1 \pm 1.3$ & $1.7 \pm 1.9$ & $3.3 \pm 1.7$ & $2.1 \pm 1.9$ \\
Urine RBC (RBCs/HPF) & $>100$ & $>100$ & $>100$ & $>100$ \\
Cellular crescent (\%) & $19.0 \pm 19.2$ & $30.5 \pm 26.6$ & $6.9 \pm 9.9$ & $4.1 \pm 5.8$ \\
Fibrocellular crescent (\%) & $14.7 \pm 17.6$ & $35.1 \pm 37.3$ & $21.1 \pm 23.4$ & $10.1 \pm 11.4$ \\
Fibrous crescent $(\%)$ & $12.7 \pm 18.7$ & $0.0 \pm 0.0$ & $3.9 \pm 6.3$ & $5.3 \pm 11.0$ \\
\hline
\end{tabular}

ANCA, anti-neutrophil cytoplasmic antibody; GN, glomerulonephritis; GBM, glomerular basement membrane; HSPN, Henoch-Schönlein purpura nephritis; BP, blood pressure; Cr, creatinine; RBC, red blood cell; $\mathrm{HPF}$, high power fields

Data of age, systolic $\mathrm{BP}$, diastolic $\mathrm{BP}$, serum $\mathrm{Cr}$, and urine protein is shown as mean $\pm \mathrm{SD}$

Data of urine RBC is shown as mean urine RBCs/HPF

Percentage of cellular, fibrocellular, or fibrous crescents is calculated as the number of glomeruli with crescents/total glomeruli

dation of fibrin and rupture of the GBM were observed in necrotizing lesions (Fig. 2I). In anti-GBM GN, multiple ruptures of the GBM were noted with fibrin exudation and the formation of cellular crescents (Fig. 2B). Multiple ruptures of CD34-positive glomerular capillaries were seen, as were a loss of glomerular endothelial cells and fibrin exudation (Fig. 2F). Cellular crescents were accompanied by the infiltration of a few leukocytes in glomerular capillaries and fibrin exudation (Fig. 2J). In lupus GN and HSPN with high activity, global (lupus GN) or segmental (HSPN) endocapillary proliferative lesions had developed with inflammatory cell infiltration (Fig. 2C and D). In part of some lesions, necrosis had occurred with exudation of fibrin and rupture of the GBM that led to cellular crescent formation. In endocapillary proliferative lesions, the CD34-positive cells had disappeared, but inflammatory cell infiltration was present (Fig. 2G and $\mathbf{H})$. In cases of lupus GN and HSPN, glomerular capillaries could not be detected, but infiltrating cells and fibrin exudation were observed (Fig. 2K and L).

CD34+ Glomerular Capillaries in the Acute Active and Chronic Phases in Crescentic GN

During the progression of necrotizing glomerular lesions in the acute and active phase to sclerotic or collaptic glomerular lesions in the chronic phase, cellular crescents were developed to form fibrous crescents through fibrocellular crescents.

In the glomeruli with cellular crescents, glomerular necrotizing lesions were noted, with rupture of the GBM and disappearance of CD34+ endothelial cells (Fig. 3A and B). In the electron microscopic findings, the endothelial cells disappeared in the glomerular capillaries with fibrin exudation (Fig. 3C). In the glomeruli with fibrocellular crescents (Fig. 3D and E), CD34+ cells could not be detected in the progressive sclerotic lesions in glomeruli. The CD34+ capillaries without necrosis had a tendency to collapse, but CD34+ cells remained. By electron microscopy, it was noted that the glomerular capillaries were not found in the sclerotic lesions in glomeruli with extracellular matrix accumulation and the formation of fibrocellular crescents (Fig. 3F).

Glomerular sclerosis developed in the glomeruli with fibrous crescents (Fig. 4A). CD34+ cells and endothelial cells could not be detected in the sclerotic lesions in the electron microscopic images (Fig. 4B and C). This suggested that glomerular sclerosis developed without capillary regeneration after capillary injury. In the collaptic glomeruli, collapse of the glomerular capillaries was noted in the glomeruli that avoided the development of necrotizing lesions, with wrinkling of the GBM and disappearance of CD34+ cells (Fig. 4D and E). The glomerular endothelial cells disappeared in the collapsed glomeruli with wrinkling of the GBM (Fig. 4F).

These findings suggested that immunohistochemical staining for CD34 was a useful technique to detect glomerular endothelial cells and glomerular capillaries morphologically. Furthermore, glomerular capillary injury and the loss of glomerular endothelial cells was associated with the formation of necrotizing and crescentic lesions and their progression in crescentic GN (Table 2). 

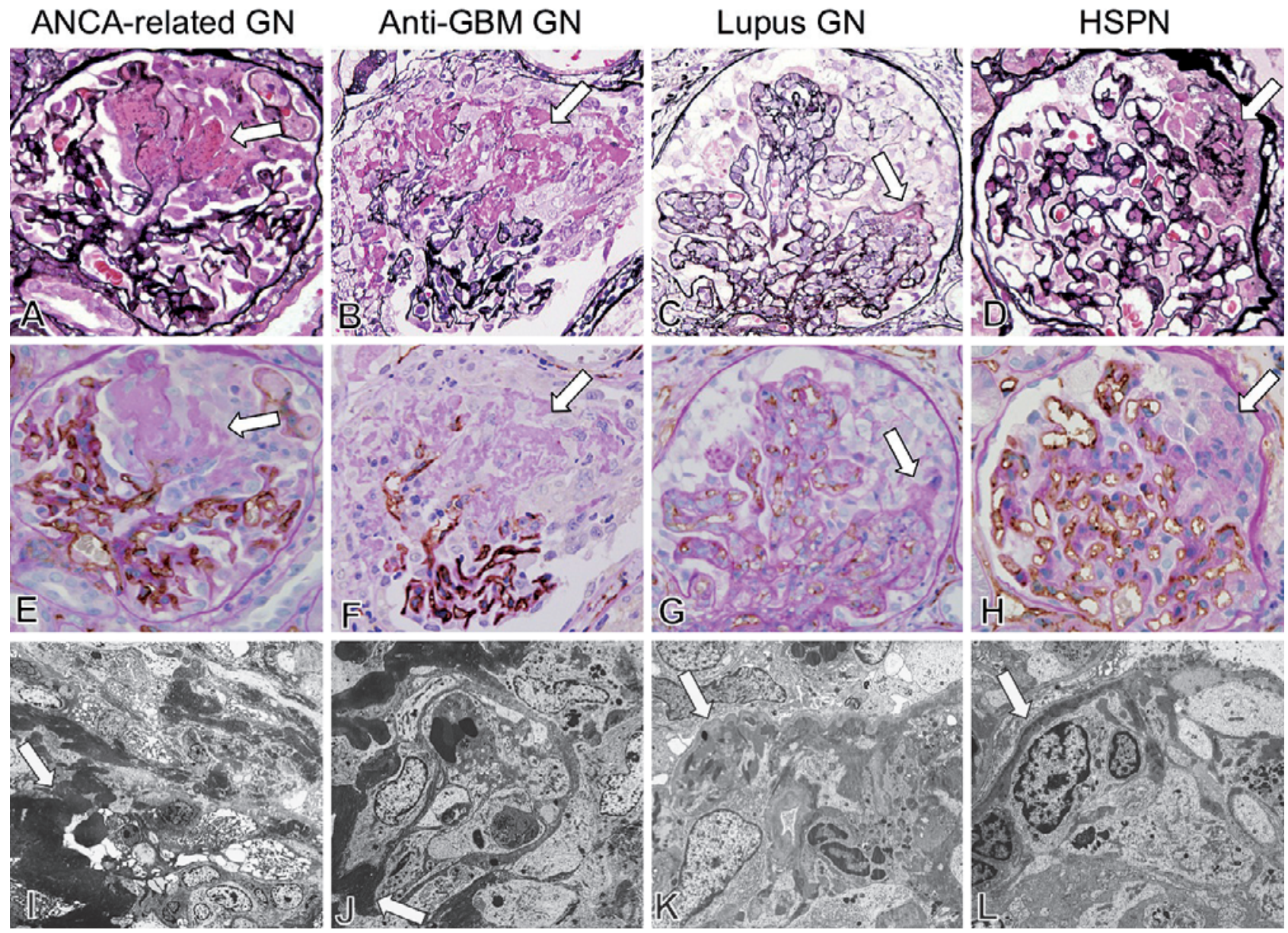

Fig. 2 The glomerular capillary injury in cases of crescentic glomerulonephritis (GN)

PAM staining (A-D: $\times 600)$, CD34 staining $(\mathrm{E}-\mathrm{H}: \times 600)$, and electron microscopic findings $(\mathrm{I}: \times 6,000 ; \mathrm{J}-\mathrm{L}: \times 10,000)$ in pauci-immune ANCA-associated GN (A, E, L), anti-GBM GN (B, F, J) and immune-complex type GN, such as lupus GN (C, G, K) and Henoch-Schönlein purpura nephritis (HSPN) (D, H, L). Focal necrotizing glomerular lesions (arrow in A to D) were found with rupture of the glomerular basement membrane (GBM), loss of CD34+ endothelial cells and fibrin exudation (arrow in E to H). Global or segmental endocapillary proliferative lesions were noted with cellular infiltrates in the immune-complex type GN (lupus GN and HSPN); however, no obvious cellular infiltrates was seen in ANCA-associated GN or anti-GBM GN. In the electron microscopy studies, the exudation of fibrin (arrow in I to L) were observed in necrotizing lesions.

\section{Discussion}

The present study demonstrated that immunohistochemical staining for CD34 could detect the glomerular capillary network morphologically in normal and diseased glomeruli in formalin-fixed paraffin-embedded routine renal biopsy tissue sections. By using CD34 immunostaining, it was easy to identify the loss of glomerular capillaries with endothelial cell injury in crescentic GN.

Furthermore, the loss of glomerular capillaries with endothelial cell injury was strongly associated with the formation of necrotizing and crescentic glomerular lesions in crescentic GN, even in the pauci-immune type GN, anti-GBM GN and immune-complex type GN. In addition, impaired capillary regeneration with a loss of endo- thelial cells contributed to the development of glomerular collapse and sclerosis in crescentic GN.

In the present study, we employed immunohistochemical staining for CD34 to detect the morphological alterations of glomerular capillaries in normal and diseased glomeruli because we decided that it provided the best detection of glomerular capillaries using our techniques among the various endothelial cell-associated proteins which we examined, including CD34, CD31, vWF, thrombomodulin, CD146, caveolin-1 and nestin.

CD34, a $115 \mathrm{kD}$ membrane glycoprotein, is a ligand for L-selectin and is known to be expressed on vascular endothelial cells ${ }^{9-11}$. In the kidney, CD34 is sometimes employed as an endothelial cell marker for glomerular capillaries and peritubular capillaries, and some previous studies have demonstrated that a loss of CD34+ capillar- 


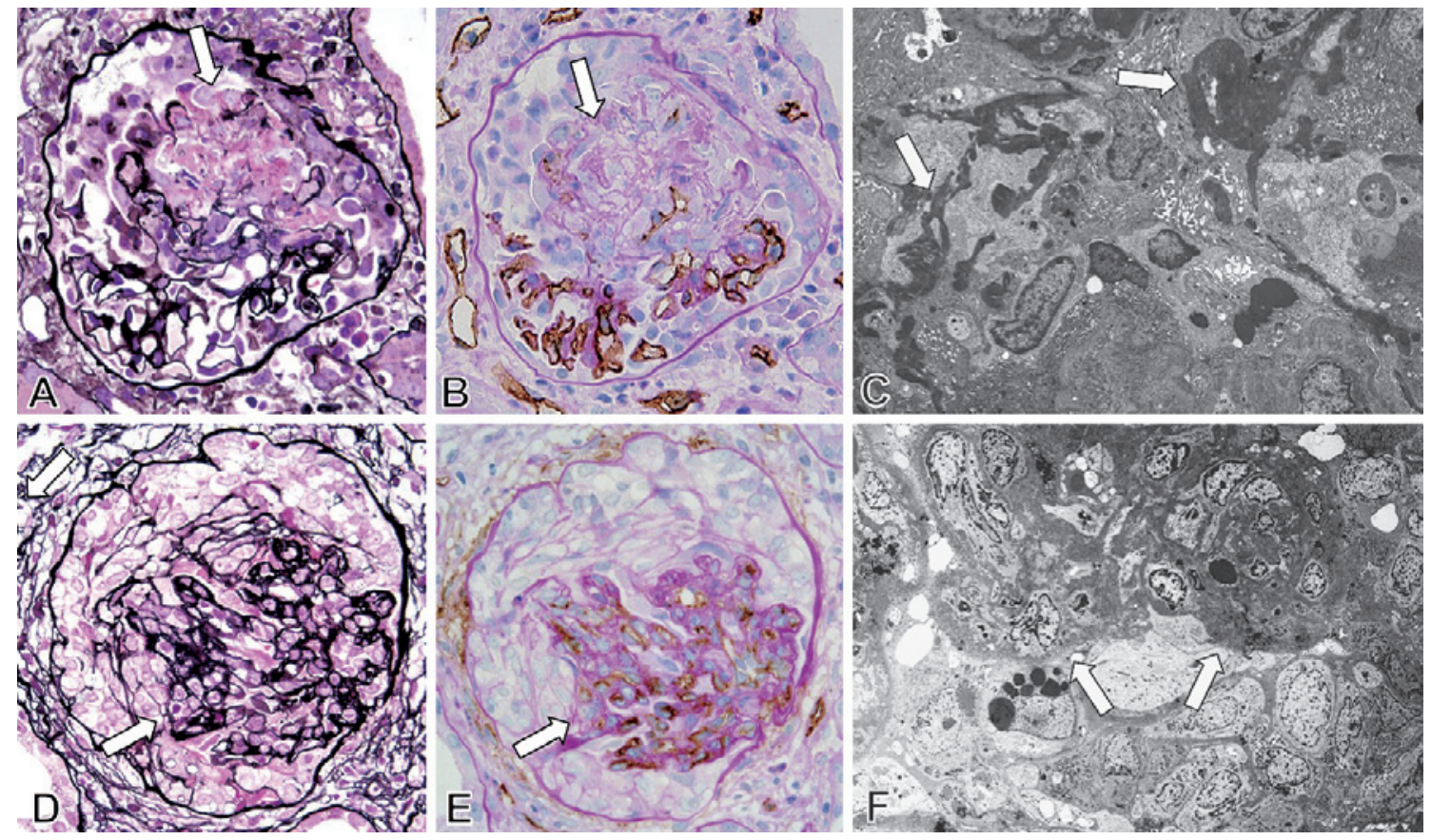

Fig. 3 Glomeruli with the formation of cellular and fibrocellular crescents

PAM staining (A, D: $\times 600)$, CD34 staining (B, E: $\times 600)$, and electron microscopic findings (C: $\times 10,000 ; \mathrm{F}: \times 7,000)$ in the glomeruli with cellular $(\mathrm{A}-\mathrm{C})$ and fibrocellular $(\mathrm{D}-\mathrm{F})$ crescents.

The formation of cellular crescents was associated with glomerular necrotizing lesions (arrow in A and B) and rupture of the GBM. CD34+ cells remained outside the necrotizing lesion, but disappeared in the necrotic lesion itself. Glomerular capillaries could not be noted in necrotizing lesions with fibrin exudation (arrow in C). Necrotizing glomerular lesions with cellular crescents gradually progressed to proliferative and sclerotic lesions (arrow in D to F) with fibrocellular crescents. CD34+ glomerular capillaries were not found in sclerotic lesions in the glomeruli with the accumulation of extracellular matrix and the formation of fibrocellular crescents.

ies in the glomeruli and interstitium is associated with the development of glomerular sclerosis and interstitial fibrosis, respectively ${ }^{12,13}$. In addition, CD34 is expressed on the surface of lymphohematopoietic stem and progenitor cells, bone marrow stromal cells and embryonic and immature fibroblasts ${ }^{9-11}$. CD34 is also known to be one of the makers of endothelial progenitor cells in peripheral blood and from bone marrow, and several studies have demonstrated that endothelial progenitor cells in the peripheral blood play an important function in vasculogenesis and acquire the ability to induce vascular repair after vascular injury ${ }^{14,15}$. In clinical setting, the defective vasculogenesis through impaired endothelial precursor cells is thought to play a role in the pathogenesis of systemic sclerosis, and circulating endothelial precursors could be a target for therapeutic strategies for collagen disease $\mathrm{e}^{16,17}$.

In the kidneys, bone marrow cells and endothelial progenitor cells were both shown to be involved in the repair of injured glomerular capillaries in experimental $\mathrm{GN}^{18,19}$. Therefore, immunostaining for CD34 may be beneficial for the detection of remaining glomerular cap- illaries, as well as for repairing glomerular capillaries after injury in GN.

The results of recent studies suggest that the renal microvasculature plays a major role in maintaining the hemodynamics and renal function, and that injury to the renal microvasculature is a crucial determinant of the progressive deterioration of renal function, as well as the progression of renal diseases ${ }^{20-24}$. Glomerular capillary and endothelial injury plays an important role in the pathogenesis of GN and is viewed as a crucial factor in disease progression in patients with glomerular sclerosis and renal dysfuntion ${ }^{25,26}$. Our previous studies using an anti-GBM GN model demonstrated that the initiation and progression of crescentic GN is associated with the destruction of the capillary network in the necrotizing glomerular lesions ${ }^{27}$. In addition, impairment of the glomerular capillary repair after injury and capillary regression also contributes to the development of glomerular sclerosis. Based on these results, we concluded that the progression of crescentic GN with renal dysfunction is determined by the severity of the injury to the glomerular capillary walls and impaired repair after in- 

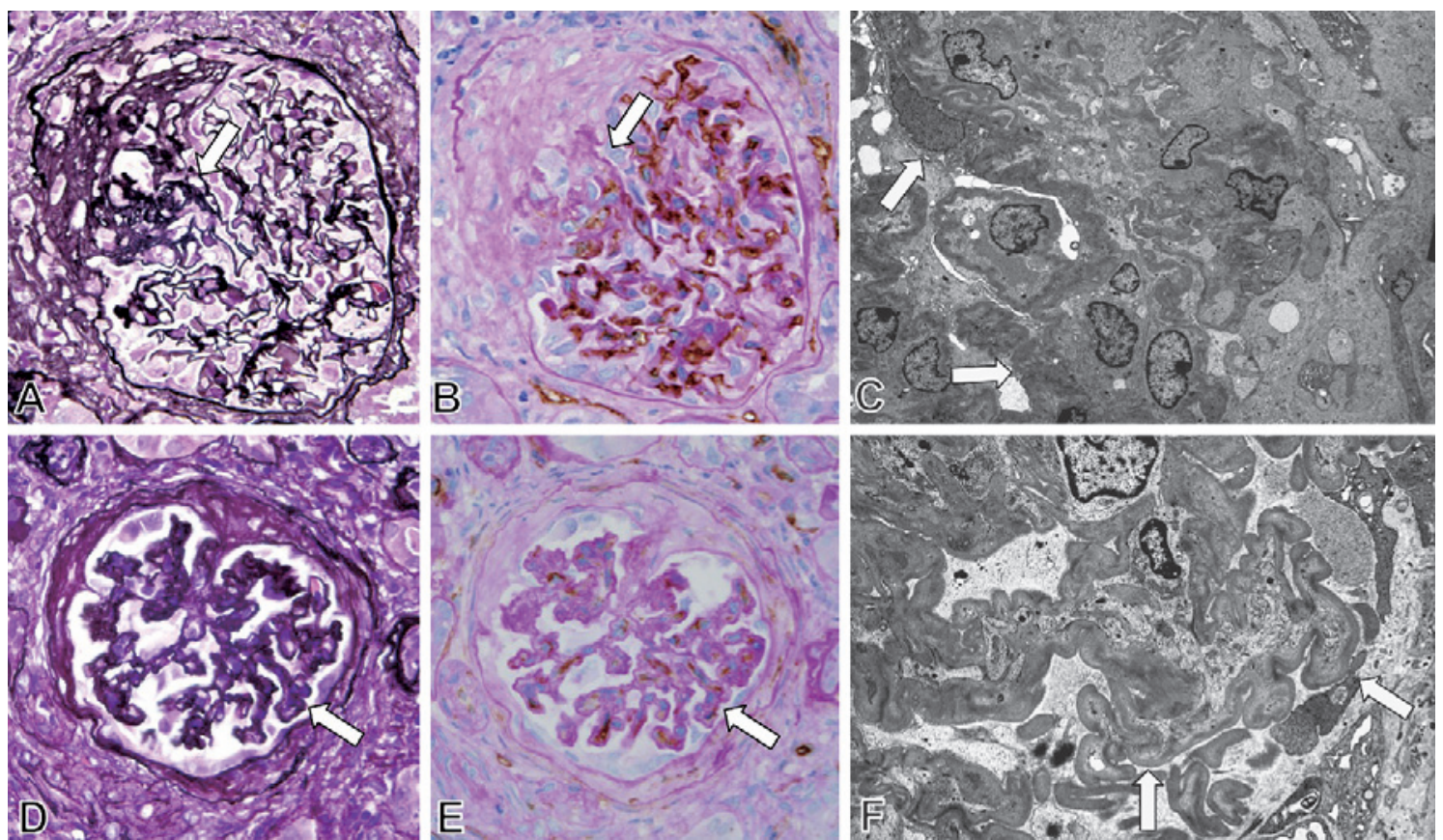

Fig. 4 Chronic glomerular lesions in crescentic GN

PAM staining (A, D: $\times 600)$, CD34 staining $(B, E: \times 600)$, and electron microscopic findings $(C, F: \times 10,000)$ in the glomeruli with segmental sclerosis and fibrous crescent $(A-C)$ and collapsed glomeruli $(D-F)$.

Glomerular sclerosis with fibrous crescents and glomerular collapse developed as chronic glomerular lesions after necrotizing and cellular crescentic glomerular lesions. In sclerotic lesions (arrow in A to C), the glomerular capillary networks could not be detected by PAM staining and CD34 immunostaining or in the electron microscopic findings. Glomerular endothelial cells disappeared in collapsed glomeruli (arrow in D to F) with wrinkling of the GBM in the PAM staining, CD34 immunostaining, and electron microscopic findings.

Table 2 The percent area of lesions in glomeruli

Glomeruli with cellular crescents

\begin{tabular}{lcccc}
\hline & ANCA-associated GN & Anti-GBM GN & Lupus GN & HSPN \\
\hline necrotizing lesion (\%) & $12.6 \pm 9.2$ & $46.3 \pm 32.5$ & $10.9 \pm 6.5$ & $9.8 \pm 5.3$ \\
lack of CD34-staining area (\%) & $15.2 \pm 7.5$ & $50.9 \pm 18.4$ & $38.3 \pm 17.4$ & $16.6 \pm 11.1$ \\
crescentic lesion (\%) & $45.2 \pm 21.1$ & $72.8 \pm 21.2$ & $22.7 \pm 10.2$ & $20.1 \pm 11.9$ \\
\hline
\end{tabular}

Glomeruli with fibrocellular and fibrous crescents

\begin{tabular}{lccrc}
\hline & ANCA-associated GN & Anti-GBM GN & Lupus GN & HSPN \\
\hline sclerotic lesion (\%) & $24.3 \pm 17.9$ & $62.1 \pm 40.4$ & $20.1 \pm 13.6$ & $24.5 \pm 23.9$ \\
lack of CD34-staining area (\%) & $26.9 \pm 15.2$ & $67.5 \pm 43.2$ & $24.8 \pm 21.7$ & $26.5 \pm 19.7$ \\
crescentic lesion (\%) & $38.8 \pm 15.9$ & $80.7 \pm 28.4$ & $36.1 \pm 18.3$ & $29.7 \pm 21.6$ \\
\hline
\end{tabular}

The percent areas in glomerulus with crescent were evaluated for the area of necrotizing glomerular lesion, the area of sclerotic glomerular lesion, the area of lack of CD34 staining, and the area of crescentic lesions. ANCA, anti-neutrophil cytoplasmic antibody; GN, glomerulonephritis; GBM, glomerular basement membrane; HSPN, Henoch-Schönlein purpura nephritis

jury.

The present study using clinical renal biopsies also demonstrated that crescentic GN was accompanied by the destruction and loss of the CD34+ glomerular capillaries with fibrin exudation, indicating that the loss of glomerular endothelial cells and rupture of the GBM in necrotizing lesions might mediate crescentic GN. Glomerular crescent formation was a common finding after the occurrence of necrotizing glomerular lesions with GBM rupture, even in cases with the pauci-immune type GN, anti-GBM GN and immune-complex type of GN. Furthermore, glomerular necrotizing lesions progressed 
to collaptic or sclerotic glomerular lesions that were associated with the loss of glomerular capillaries and endothelial cells, with the accumulation of extracellular matrix, thus indicating that incomplete repair of glomerular capillaries after injury was associated with the development of glomerular collapse and sclerosis. In the clinical setting, crescentic GN is known to be one of the most active and severe types of $\mathrm{GN}^{1-4}$. Our results and those of previous studies indicate that glomerular capillary injury with glomerular endothelial cell injury might mediate the necrotizing and crescentic glomerular lesions and their progression to glomerular sclerosis in crescentic GN.

Our previous studies using experimental GN models demonstrated that complete capillary repair in damaged glomeruli can lead to full recovery of the glomerular architecture with resolution of $\mathrm{GN}^{28-31}$. In experimental necrotizing and crescentic anti-GBM GN, the augmentation of angiogenic glomerular capillary repair by the administration of vascular endothelial cell growth factor (VEGF) mediated the resolution of glomerular crescents, as well as the progression of crescentic $\mathrm{GN}^{32}$. We have considered that angiogenic capillary repair is a crucial event to allow for glomerular healing, as well as recovery from GN. We believe that the development of necrotizing and crescentic GN may therefore be both prevented and resolved by treatment utilizing stimulating angiogenic capillary repair.

\section{Conclusions}

Immunohistochemical staining for CD34 is thus considered to be useful to observe the glomerular capillary injuries in conventional formalin-fixed paraffin-embedded renal biopsy tissue sections. Severe glomerular capillary and endothelial cell injury was commonly associated with necrotizing and crescentic glomerular lesions in all forms of crescentic GN, such as pauci-immune type GN, anti-GBM GN and immune-complex type GN.

Conflict of Interest: The authors have no conflicts of interest to declare in association with this study.

Acknowledgements: We express special thanks to Mr. Takashi Arai, Ms. Mitue Kataoka, Ms. Kyoko Wakamatsu, Ms. Arimi Ishikawa and Ms. Naomi Kuwahara for the expert technical assistance. We are also grateful to Drs. Yukinari Masuda, Shinya Nagasaka, Toru Igarashi, Tsuyoshi Yanagihara, Yukinao Sakai, Takehisa Yamada and Ryuji Ohashi for their useful advice.

\section{References}

1. Jennette JC, Thomas DB: Crescentic glomerulonephritis. Nephrol Dial Transplant 2001; 16 (Suppl 6): 80-82.

2. Moroni G, Ponticelli C: Rapidly progressive crescentic glomerulonephritis: Early treatment is a must. Autoimmun Rev 2014; 13: 723-729.

3. Bajema IM: Pathological classification of anti-neutrophil cytoplasmic antibody (ANCA)-associated glomerulonephritis. Clin Exp Immunol 2011; 164 (Suppl 1): 14-16.

4. Jennette JC, Falk RJ: The role of pathology in the diagnosis of systemic vasculitis. Clin Exp Rheumatol 2007; 25 (Suppl 44): S52-S56.

5. Michiels C: Endothelial cell functions. J Cell Physiol 2003; 196: 430-443.

6. Endemann DH, Schiffrin EL: Endothelial dysfunction. J Am Soc Nephrol 2004; 15: 1983-1992.

7. Fujita E, Shimizu A, Kaneko T, et al.: Proliferative glomerulonephritis with monoclonal immunoglobulin G3א deposits in association with parvovirus B19 infection. Hum Pathol 2012; 43: 2326-2333.

8. Mii A, Shimizu A, Kaneko T, et al.: Renal thrombotic microangiopathy associated with chronic graft-versus-host disease after allogeneic hematopoietic stem cell transplantation. Pathol Int 2011; 61: 518-527.

9. Lanza F, Healy L, Sutherland DR: Structural and functional features of the CD34 antigen: an update. J Biol Regul Homeost Agents 2001; 15: 1-13.

10. Krause DS, Fackler MJ, Civin CI, May WS: CD34: structure, biology, and clinical utility. Blood 1996; 87: 1-13.

11. Fina L, Molgaard HV, Robertson D, et al.: Expression of the CD34 gene in vascular endothelial cells. Blood 1990; 75: 2417-2426.

12. Ishii $Y$, Sawada T, Kubota K, Fuchinoue S, Teraoka S, Shimizu A: Injury and progressive loss of peritubular capillaries in the development of chronic allograft nephropathy. Kidney Int 2005; 67: 321-332.

13. Gluhovschi C, Gluhovschi G, Potencz E, et al.: The endothelial cell markers von Willebrand Factor (vWF), CD31 and CD34 are lost in glomerulonephritis and no longer correlate with the morphological indices of glomerular sclerosis, interstitial fibrosis, activity and chronicity. Folia Histochem Cytobiol 2010; 48: 230-236.

14. Kawamoto A, Asahara T: Role of progenitor endothelial cells in cardiovascular disease and upcoming therapies. Catheter Cardiovasc Interv 2007; 70: 477-484.

15. Mackie AR, Losordo DW: CD34-positive stem cells: in the treatment of heart and vascular disease in human beings. Tex Heart Inst J 2011; 38: 474-485.

16. Kuwana M, Okazaki Y, Yasuoka H, Kawakami Y, Ikeda Y: Defective vasculogenesis in systemic sclerosis. Lancet 2004; 364: 603-610.

17. Kuwana M, Okazaki Y: Brief report: impaired in vivo neovascularization capacity of endothelial progenitor cells in patients with systemic sclerosis. Arthritis Rheumatol 2014; 66: 1300-1305.

18. Li B, Morioka T, Uchiyama M, Oite T: Bone marrow cell infusion ameliorates progressive glomerulosclerosis in an experimental rat model. Kidney Int 2006; 69: 323-330.

19. Reinders ME, Rabelink TJ, Briscoe DM: Angiogenesis and endothelial cell repair in renal disease and allograft rejection. J Am Soc Nephrol 2006; 17: 932-942.

20. Bohle A, Mackensen-Haen S, Wehrmann M: Significance of postglomerular capillaries in the pathogenesis of chronic renal failure. Kidney Blood Press Res 1996; 19: 
191-195.

21. Shimizu A, Yamada K, Sachs DH, Colvin RB: Persistent rejection of peritubular capillaries and tubules is associated with progressive interstitial fibrosis. Kidney Int 2002; 61: 1867-1879.

22. Ohashi R, Shimizu A, Masuda Y, et al.: Peritubular Capillary Regression during the Progression of Experimental Obstructive Nephropathy. J Am Soc Nephrol 2002; 13: 1795-1805.

23. Kang DH, Kanellis J, Hugo C, et al.: Role of the microvascular endothelium in progressive renal disease. J Am Soc Nephrol 2002; 13: 806-816.

24. Nangaku M: Mechanisms of tubulointerstitial injury in the kidney: final common pathways to end-stage renal failure. Intern Med 2004; 43: 9-17.

25. Shimizu A, Yamada K, Sachs DH, Colvin RB: Mechanisms of chronic renal allograft rejection. II. Progressive allograft glomerulopathy in miniature swine. Lab Invest 2002; 82: 673-686.

26. Yamanaka N, Shimizu A: Role of glomerular endothelial damage in progressive renal disease. Kidney Blood Press Res 1999; 22: 13-20.

27. Shimizu A, Kitamura H, Masuda Y, Ishizaki M, Sugisaki $\mathrm{Y}$, Yamanaka N: Rare glomerular capillary regeneration and subsequent capillary regression with endothelial cell apoptosis in progressive glomerulonephritis. Am J Pathol 1997; 151: 1231-1239.
28. Masuda Y, Shimizu A, Kataoka M, et al.: Inhibition of capillary repair in proliferative glomerulonephritis results in persistent glomerular inflammation with glomerular sclerosis. Lab Invest 2010; 90: 1468-1481.

29. Shimizu A, Masuda Y, Kitamura H, Ishizaki M, Sugisaki Y, Yamanaka N: Recovery of damaged glomerular capillary network with endothelial cell apoptosis in experimental proliferative glomerulonephritis. Nephron 1998; 79: 206-214.

30. Mori T, Shimizu A, Masuda Y, Fukuda Y, Yamanaka N: Hepatocyte growth factor-stimulating endothelial cell growth and accelerating glomerular capillary repair in experimental progressive glomerulonephritis. Nephron Exp Nephrol 2003; 94: e44-54.

31. Masuda Y, Shimizu A, Mori T, et al.: Vascular endothelial growth factor enhances glomerular capillary repair and accelerates resolution of experimentally-induced glomerulonephritis. Am J Pathol 2001; 159: 599-608.

32. Shimizu A, Masuda Y, Mori T, et al.: Vascular Endothelial Growth Factor165 Resolves Glomerular Inflammation and Accelerates Glomerular Capillary Repair in Rat Anti-GBM glomerulonephritis. J Am Soc Nephrol 2004; 15: 26552665.

(Received, September 9, 2014)

(Accepted, December 10, 2014) 